\title{
Lebenssituation und Perspektiven junger Menschen ohne festen Wohnsitz
}

\section{Ergebnisse und Reflexion einer quantitativen Studie}

\author{
Sarah Beierle $\cdot$ Carolin Hoch
}

Eingegangen: 10. Juni 2021 / Angenommen: 12. Oktober 2021 / Online publiziert: 10. November 2021 (C) Der/die Autor(en) 2021

Zusammenfassung Der Beitrag stellt zentrale Ergebnisse einer Befragung vor, in der versucht wurde, die Lebenssituation von wohnungslosen oder obdachlosen Jugendlichen und jungen Erwachsenen in drei deutschen Großstädten quantitativ zu erheben. Die Wohnsituation von ,Straßenjugendlichen“ scheint dadurch, dass viele Betroffene zwischenzeitlich immer wieder bei Freunden oder Bekannten unterkommen, in erheblichem Maße durch Wohnungslosigkeit geprägt. Das Durchschnittsalter des Eintritts in eine Straßenkarriere liegt bei 16 bis 17 Jahren. Die befragten Jugendlichen sind von multiplen, insbesondere familiären Problemlagen betroffen, geben an, nicht freiwillig ohne festen Wohnsitz zu sein und sind mit ihrer Lebenssituation überwiegend unzufrieden. Dennoch sehen sie optimistisch in die Zukunft und glauben, mittelfristig ihre Wohnungs- bzw. Obdachlosigkeit überwinden zu können. Die Daten weisen außerdem darauf hin, dass junge volljährige Menschen ohne festen Wohnsitz vorwiegend mit dem Jobcenter in Kontakt stehen und das Jugendamt nicht mehr als relevantes Hilfesystem in Erscheinung tritt.

Schlüsselwörter Straßenkarrieren · Wohnungslosigkeit · Obdachlosigkeit · Hilfesystem · Lebenslagen Straßenjugendlicher

\footnotetext{
Sarah Beierle $(\square) \cdot$ Carolin Hoch

Außenstelle Halle, Deutsches Jugendinstitut, Franckeplatz 1, Haus 12/13, 06110 Halle, Deutschland E-Mail: beierle@dji.de

Carolin Hoch

E-Mail: hoch@dji.de
} 


\title{
Life situation and perspectives of young people without permanent housing
}

Results and reflection of a quantitative study

\begin{abstract}
This article presents key findings from a survey to quantitatively assess the living situation of adolescents and young adults in three major German cities who are homeless or have no permanent residence. The housing situation of "street youth" is characterized to a greater extent by homelessness where many of those affected repeatedly find temporary shelter with friends or acquaintances. The average age at which these individuals start living on the street is 16 to 17 years. The young people interviewed are affected by multiple problems, especially family problems, state that they are not voluntarily without a permanent residence, and are predominantly dissatisfied with their living situation. Nevertheless, they are optimistic about the future and believe they will be able to overcome their homelessness or lack of permanent housing in the medium term. The data also indicate that young adults without a permanent home are mainly in contact with the job center and that the youth welfare office is no longer a relevant support system.
\end{abstract}

Keywords Street careers $\cdot$ Homelessness $\cdot$ Without permanent residence $\cdot$ Support system $\cdot$ Living conditions of street youth

Eltern oder ersatzweise die Jugendhilfe sind dafür zuständig, Kindern und Jugendlichen einen sicheren Wohnort zu bereiten. Ein fester Wohnraum stellt aus sozialpsychologischer Perspektive einen Rückzugsort dar, um sich zeitweise aus der Gesellschaft zurückzuziehen und in Privatsphäre ungestört und geschützt schlafen, sich erholen oder entspannen zu können (Häußermann und Siebel 2000). Dabei spielt weniger die Größe des territorialen Ausschnitts eine Rolle, als vielmehr die Möglichkeit, sich diesen zu eigen zu machen und gestalten zu können (BrändleStöh 1999). Abhängig vom kulturellen und sozioökonomischen Kontext reicht ein solcher Ort im Kindesalter vom eigenen Bett, über einen eigenen Bereich bis hin $\mathrm{zu}$ einem eigenen Zimmer. Mehr noch als an die faktische Wohnraumsituation ist das Wohlbefinden am Wohnsitz aber an die Beziehung zu den im Haushalt lebenden Personen gebunden.

Spätestens mit Erreichen des Jugendalters nimmt die Auseinandersetzung mit der eigenen aktuellen Wohnsituation zu. Es werden in Abhängigkeit mit dem gefühlten Wohlbefinden, den monetären Ressourcen und den (beruflichen) Plänen Vorstellungen von einem Wohnen außerhalb der elterlichen Wohnung oder einer Wohneinrichtung der Jugendhilfe entwickelt. Für die Realisierung eines Auszugs lag das Durchschnittsalter des Auszugs aus dem Elternhaus 2019 bei 23,7 Jahren, wobei junge Frauen etwas früher als junge Männer einen eigenen Hausstand gründen (Destatis 2020).

Trotz eines ausdifferenzierten Hilfesystems gibt es in Deutschland Minderjährige und junge Erwachsene, die wohnungs- oder obdachlos sind. Sie sind aus unsicheren und für sie unhaltbaren Wohnverhältnissen geflüchtet, mussten den vorherigen 
Wohnsitz verlassen oder sind gar „rausgeschmissen“ worden. Es ist ihnen nicht gelungen, rechtzeitig neue sichere Wohnverhältnisse zu realisieren.

Der vorliegende Beitrag nimmt die Lebenssituation von Jugendlichen und jungen Erwachsenen, die ohne festen Wohnsitz sind oder sich auf unbestimmte Zeit abseits ihres Wohnsitzes aufhalten, in den Blick. Ein besonderer Fokus wird dabei auf die jungen Erwachsenen im Alter von 18 bis einschließlich 24 Jahren geworfen. In diesem Alter sind vielfältige Herausforderungen zu meistern, wie z. B. Peerund Partnerbeziehungen aufzubauen, berufliche Zukunftspläne und Ideen für eine eigenständige Lebensführung zu entwickeln (Bundesministerium für Familie, Senioren, Frauen und Jugend 2017; Hurrelmann und Quenzel 2015). Den meisten jungen Menschen gelingt dies gut - insbesondere auch, weil sie auf individuelle und familiäre Ressourcen zurückgreifen können. Diejenigen, die nicht über die entsprechenden Voraussetzungen verfügen (z.B. wegen ihrer sozialen Herkunft oder ihrer Bildungsbiografie) profitieren nicht von diesen neugewonnenen Freiheitsgraden (Reißig 2019).

Nachfolgend werden zunächst die zentralen Herausforderungen in der Forschung zu jungen Menschen ohne festen Wohnsitz dargestellt. Anschließend werden Fragestellungen abgeleitet, die auf Grundlage einer quantitativen Erhebung beantwortet werden sollen. Es folgt ein Fazit, in dem die Möglichkeiten und Grenzen der quantitativen Studie erörtert werden.

\section{Herausforderungen in der Forschung zu jungen Menschen ohne festen Wohnsitz}

\subsection{Unterschiedliche Begriffsverwendungen}

Straßenkinder, Straßenjugendliche, junge Menschen ohne festen Wohnsitz, obdachlose Jugendliche, wohnungslose Jugendliche, nicht sesshafte Jugendliche, Ausreißer, Trebegänger, Trebern, Crash Kids oder DropOuts (Mögling et al. 2015, S. 10) dies sind nur einige Bezeichnungen für ein ähnliches Phänomen, nämlich für junge Menschen (Kinder, Jugendliche und junge Erwachsene) in prekären Lagen, die ohne festen Wohnsitz sind, auf der Straße leben oder irgendwo behelfsmäßig untergekommen sind. Bei der Verwendung dieser Begriffe ergeben sich mehrere Problematiken.

Teilweise werden die Begriffe synonym verwendet und sollen auf ein und dasselbe Problem verweisen, wecken jedoch ganz unterschiedliche Assoziationen, zum Beispiel, dass das Phänomen nur auf bestimmte Personen begrenzt sei. Spricht man von Kindern, Jugendlichen oder jungen Menschen, dann werden - gewollt oder ungewollt - Altersgruppen festgelegt und andere Altersgruppen außen vor gelassen. Außerdem kann der Eindruck entstehen, dass nur Problemlagen bestimmter Gruppen, z. B. von Obdachlosen, die ausschließlich und ohne festen Wohnsitz auf der Straße leben, gemeint sind.

Aber auch, wenn die Begriffe bewusst für eine bestimmte Teilgruppe des Phänomens verwendet werden, ergeben sich Unschärfen. So können beispielsweise die Begriffe „obdach- und wohnungslos“ als Synonyme verwendet werden oder aber als voneinander abgegrenzte Begriffe (Frietsch und Holbach 2016, S. 13). Hierbei 
wird als „obdachlos“ das gänzliche Fehlen einer Unterkunft und das ausschließliche Leben auf der Straße verstanden. Im Falle von ,,wohnungslos“ kann man irgendwo untergekommen sein, bei Freunden oder in Behelfsunterkünften.

Heute wird zumeist die Bezeichnung „Straßenjugendliche“ gegenüber dem zuvor stärker geläufigen Begriff „Straßenkinder“ vorgezogen. Beide Begriffe - sowohl „Straßenkinder“"als auch „Straßenjugendliche“ - nehmen mit ihrem Bezug zur „Straße“ eine klare Verortung vor. In den allermeisten Fällen sind die Begriffe jedoch nicht auf die Straße als Hauptaufenthaltsort begrenzt, sondern schließen alle vorübergehenden und behelfsmäßigen Wohngelegenheiten fernab eines festen Wohnsitzes mit ein. Jedoch weckt die Bezeichnung den Anschein, dass nur von obdachlosen jungen Menschen die Rede ist, die ohne jegliche Unterkunft auf der Straße leben.

Von Kritikern wird zudem eine leichtfertige Rhetorik im Zusammenhang mit der Zielgruppe angemahnt. Es werde dabei zu wenig reflektiert, wie sehr die Wortwahl das Verständnis und den Umgang mit dem angesprochenen sozialen Phänomen vorherbestimmt und unter Umständen sogar deformiert (Liebel 2018). So würden die Bezeichnungen „Straßenkinder“ oder „Straßenjugendlicher“ suggerieren, dass die Lebenssituation der Betroffenen statisch und somit unveränderbar sei, was zu einem Labelling und einer Stigmatisierung Betroffener führen würde.

Dass die Lebenssituation und Aufenthaltsorte aber nicht statisch, sondern vielmehr von häufigen Wechseln geprägt sind, hat eine Forschergruppe des Deutschen Jugendinstituts bereits in den 1990er Jahren herausgearbeitet (Permien und Zink 1998). Nach Zeiten in Obdachlosigkeit ohne jegliches Unterkommen schlossen sich häufig Phasen des zwischenzeitlichen Unterkommens an. Es wird also von Pendelbewegung zwischen dem Elternhaus, Einrichtungen, Hilfeeinrichtungen, Freunden und der Straße berichtet (Bielert 2006; Permien und Zink 1998). Für dieses Phänomen führten sie den Begriff der „Straßenkarriere“ in die Fachdebatte ein, welcher auf die Prozesshaftigkeit des Lebens ohne festen Wohnsitz verweist. Diese Prozesshaftigkeit äußert sich beispielsweise auch dadurch, dass die Abwendung von Familie, Jugendhilfeeinrichtung und Schule und die Hinwendung zur Straße als zentralen Sozialisationsort zumeist sukzessive erfolgt (Fernandez 2014, S. 326). Ebenso sukzessive würden sich Hilfeprozesse gestalten, um die Situation ohne festen Wohnsitz zu überwinden (Beierle 2017).

Dies alles verdeutlicht zentrale Hürden bei der Forschung zu jungen Menschen ohne festen Wohnsitz: Wer ist gemeint, wenn von jungen Menschen auf der Straße gesprochen wird? Welche Lebensumstände werden damit adressiert und welcher Begriff ist gerechtfertigt, um diese Personengruppe verständlich abzugrenzen?

Eine feste Definition von „Straßenjugendlichen“ oder jungen Menschen ohne festen Wohnsitz gibt es nicht und auch, welche Personen je nach Alter und Wohnsituation hinzugezählt werden, kann ganz unterschiedlich festgelegt werden. Um sich analytisch dem Phänomen annähern zu können, ist es somit notwendig, eine klare Begriffsabgrenzung vorzunehmen. Zum einen, um all die Personen adressieren zu können, die für eine Forschungsfrage relevant sind und zum anderen, um die Ergebnisse einer eindeutigen Personengruppe zuordnen zu können. 


\subsection{Erreichbarkeit der Zielgruppe und Forschungsdesign}

Neben der fehlenden Definition für die Zielgruppe gibt es weitere Aspekte, die die Betrachtung und Erforschung des Phänomens junger Menschen ohne festen Wohnsitz erschweren. Allen voran ist zu nennen, dass es sich um eine Zielgruppe handelt, die sich teils bewusst von Institutionen und Hilfeeinrichtungen fernhält und demnach als nur sehr schwer erreichbar einzuordnen ist. Hinzu kommt, dass die Personen, wie bereits beschrieben, ihren Aufenthaltsort häufig wechseln.

Im Wesentlichen gibt es zwei Möglichkeiten, um die Zielgruppe für Forschung zugänglich zu machen.

Eine erste Möglichkeit ist es, den Kontakt über typische Anlaufstellen der Betroffenen zu knüpfen. So können beispielsweise Suppenküchen, Notunterkünfte oder andere Hilfsangebote für Obdach- oder Wohnungslose genutzt werden, um Kontakt mit der Zielgruppe aufzubauen.

Eine zweite Möglichkeit besteht darin, zu versuchen, die Zielgruppe direkt anzusprechen. Allerdings sind nicht alle Personen ohne festen Wohnsitz auf diese Weise ermittelbar, denn nicht alle Obdach- oder Wohnungslosen sind durch auffällige Kleidung, gefärbte Haare, Tattoos oder Piercings zu erkennen (Fernandez 2014, S. 330). Weiterhin ist zu beobachten, dass der innerstädtische Aufenthalt von unerwünschten Gruppen (z. B. Punks) durch architektonische Konzepte und ordnungspolitische Instrumente vermehrt verhindert werden (Simon 2006, S. 162) soll und so Betroffene aus den Innenstädten und von öffentlichen Plätzen vertrieben werden.

Trotz der Bemühungen wird ein Anteil an Betroffenen durch diese Kontaktmöglichkeiten nicht erreicht werden können. Vor allem Wohnungslose, die beispielsweise bei Freunden untergekommen sind und keine oder nur sehr selten Hilfsangebote nutzen, können so nur sehr schwer oder gar nicht ausfindig gemacht werden. Es kann nur vermutet werden, wie groß die Dunkelziffer an nicht sichtbaren Betroffenen ist. Zudem ist davon auszugehen, dass bei einem Zugang über Einrichtungen eben jene Zielgruppen der Einrichtungen überrepräsentiert sind und möglicherweise spezifische Untergruppen (z.B. Migrantinnen und Migranten, junge Frauen) nicht hinreichend abgebildet werden können.

Die nur eingeschränkte Erreichbarkeit der Zielgruppe und die fehlende Definition machen es schwer, die Zielgruppe für die Forschung zugänglich zu machen. Möglich ist dabei sowohl ein qualitativer, wie auch quantitativer Zugang zur Zielgruppe. Bis dato liegen bis auf kleinere regional begrenzte quantitative Befragungen (Frietsch und Holbach 2016; Schaak 2009) fast ausschließlich qualitative Betrachtungen vor.

Qualitative Betrachtungen zeichnen sich durch eine hohe Subjektbezogenheit aus. Durch persönliche Interviews kann ein sehr genauer und detaillierter Einblick in den Alltag, die Lebensumstände und die Sinnstrukturen von Betroffenen des Samples gewonnen werden. $\mathrm{Ob}$ sich die dort gewonnenen Befunde auch bei einer großen Anzahl Befragter statistisch zeigen, wurde bislang kaum untersucht.

Weiterhin beziehen sich viele der bereits existierenden Studien nur auf ganz bestimmte Zielpersonen, z. B. nur Betroffene in Einrichtungen oder nur auf bestimmte Regionen oder einzelne Städte (Alleweldt und Leuschner 2004; Busch-Geertsema et al. 2016; Möbius 1996; Schaak 2009). Auch hier stellt sich die Frage, ob für welche Teile der „Straßenjugendlichen“ diese Ergebnisse übertragen werden können. 
Neben Befragungen sind auch andere Forschungszugänge für die Untersuchung der Zielgruppe denkbar, wie beispielsweise (teilnehmende) Beobachtungen. Um einen Einblick in den Gegenstandsbereich zu erhalten, ohne die Daten direkt bei der Zielgruppe zu ermitteln, sind außerdem Befragungen von Expertinnen und Experten (Hoch 2017) oder auch Evaluationen von Projekten, die sich mit der Zielgruppe beschäftigen möglich (Beierle 2017, 2019).

Aktuell liegt noch keine regelmäßig erstellte deutschlandweite Wohnungslosenstatistik vor. Allerdings soll es ab 2022 eine bundesweite Datenerhebung über Ausmaß und Struktur der Wohnungslosigkeit in Deutschland geben (Die Bundesregierung o.J.). Dabei werden die Daten über zwei Wege ermittelt, zum einen werden wohnungslose Menschen in Gemeinschafts- oder Notunterkünften erfasst, zum anderen soll es eine ergänzende Wohnungslosenberichterstattung über Wohnungslose geben, die bei Verwandten, Freunden oder auf der Straße leben.

\section{Datengrundlage und Fragestellungen}

Zwischen 2015 und 2016 wurde eine quantitative Befragung von jungen wohnungslosen und obdachlosen Menschen durchgeführt, um sich dem Phänomen ,Straßenjugendliche“ anzunähern (Hoch 2016). Dafür wurden Personen bis einschließlich 24 Jahren zu ihrer Wohn- und Lebenssituation befragt. Von Interesse war dabei nicht nur der aktuelle Zustand, sondern es wurden zusätzlich retrospektiv die letzten zwei Jahre abgefragt. Gefragt wurde u. a. nach ihren Aufenthaltsorten, nach den Gründen für das Leben auf der Straße, nach ihrer finanziellen Situation und danach, wie sie Hilfsangebote nutzen. Auskunft gaben sie auch dazu - und das ist vor allem im Hinblick auf die Begriffsabgrenzung eine zentrale Frage - inwieweit sie sich, in den unterschiedlichen Wohnsituationen, selbst als Straßenjugendliche empfunden haben ${ }^{1}$.

Das besondere an der Studie ist der quantitative Zugang zur Zielgruppe. Es wurde erstmalig versucht, eine Vielzahl von betroffenen jungen Menschen zum Leben ohne festen Wohnsitz zu befragen. Insgesamt konnten 316 Interviews geführt werden, von denen 297 Fälle für die Auswertung zur Verfügung standen. Die Interviews erfolgten in Berlin, Hamburg und Köln.

Um möglichst viele Betroffene interviewen zu können, wurden für die Befragung nicht nur junge Menschen angesprochen, die zum Zeitpunkt der Befragung ohne festen Wohnsitz waren, sondern es wurden auch Personen befragt, die zwar zum Zeitpunkt der Befragung einen festen Wohnsitz hatten, aber innerhalb der letzten zwei Jahre zu irgendeinem Zeitpunkt ohne festen Wohnsitz waren. Von den für die Auswertung zur Verfügung stehenden 297 Befragten hatten zum Befragungszeitpunkt 85 Personen (28,6\%) einen festen Wohnsitz, 155 Personen $(52,2 \%)$ waren wohnungslos und 57 Personen $(19,2 \%)$ obdachlos. Durch die retrospektive Erfassung der letzten zwei Jahre konnten somit ebenso vergangene Straßenepisoden erfasst werden und in die Datenauswertung mit einfließen.

\footnotetext{
1 Detaillierte Erörterungen zu Konstruktion und Inhalt des Fragebogens siehe Hoch (2016).
} 
Ein wesentliches Ziel der Untersuchung war es, sich durch die Befragung der Betroffenen einer geeigneten Definition für die Zielgruppe anzunähern. Es wurde mit einer recht breiten Arbeitsdefinition gearbeitet, um keine Gruppen im Vorhinein auszuschließen.

Unter Straßenjugendlichen verstehen wir junge Menschen [im Alter bis einschließlich 24 Jahren], die keinen festen Wohnsitz haben oder sich für eine nicht vorhersehbare Zeit abseits ihres gemeldeten Wohnsitzes (Familie oder Jugendhilfeeinrichtungen) aufhalten. Gemeint sind dabei nicht nur Jugendliche, die ausschließlich auf der Straße leben und schlafen, also gar kein Dach über dem Kopf haben, sondern auch diejenigen, die irgendwo oder bei irgendjemandem untergekommen sind, z. B. bei Freunden, in Behelfs- oder in Notunterkünften. Von Interesse sind somit alle obdach- oder wohnungslosen Jugendlichen in Deutschland. (Hoch 2017, S. 14f.) ${ }^{2}$

Um trotz der schweren Erreichbarkeit der Zielgruppe eine hinreichend große Anzahl betroffener Jugendlicher für die quantitative Erhebung gewinnen zu können, wurde der Weg über niedrigschwellige Angebote der (Straßen-)Sozialarbeit gewählt. Die Befragungen erfolgten vor allem durch Studierende der Sozialen Arbeit und Fachkräfte der Jugendsozialarbeit in den ausgewählten Einrichtungen, die hierfür extra im Rahmen des Projekts geschult wurden. Das Gros der Interviews fand im Umfeld der akquirierten Einrichtungen statt, lediglich elf Interviews erfolgten nach Ansprache der Interviewpartner/innen auf der Straße.

Bei der Interpretation und Verallgemeinerung der Ergebnisse ist zu beachten, dass es durch diesen Zugang zu Verzerrungen in den Daten gekommen sein kann, da Personen, die kaum oder gar keinen Kontakt zu Hilfeeinrichtungen haben, in der Befragungsgruppe unterrepräsentiert sind.

Folgende Fragestellungen werden nachfolgend auf Grundlage der durch die Studie generierten quantitativen Daten analysiert und mit Erkenntnissen aus der qualitativen Forschung - sofern vorliegend - abgeglichen.

1. Wo halten sich junge Menschen ohne festen Wohnsitz hauptsächlich auf?

2. Wie unterscheidet sich die Lebenssituation junger volljähriger Straßenjugendlicher gegenüber der minderjähriger Straßenjugendlicher?

3. Wie blicken die Jugendlichen in ihre Zukunft?

4. Wer versteht sich als Straßenjugendliche/r?

\section{Ergebnisse}

\subsection{Die Straßenkarrieren sind vor allem durch Wohnungslosigkeit geprägt}

Um einen Einblick in die Situation der Befragten zu erhalten, wird zunächst betrachtet, ob sie während ihrer aktuellen (für Befragte ohne festen Wohnsitz zum

\footnotetext{
2 Die Definition entstammt der 2. Projektphase der Untersuchung. Da diese im Wesentlichen jedoch nicht (außer die Altersgrenze) von der Arbeitsdefinition der 1. Projektphase abweicht, erscheint eine Verwendung an dieser Stelle als geeignet.
} 
Zeitpunkt der Befragung) oder während der letzten Straßenepisode (für Befragung mit festem Wohnsitz zum Zeitpunkt der Befragung) obdachlos oder wohnungslos waren. Mit 67,2\% (195 Personen) war der Großteil der Befragten wohnungslos. Obdachlos (gewesen) zu sein gaben 32,8\% (95 Personen) an. Sowohl bei Minderjährigen als auch bei Volljährigen zeigt sich eine ähnliche Verteilung von Obdachund Wohnungslosigkeit.

Dass die meisten der Jugendlichen nicht längerfristig ohne jegliches Obdach sind, mag im ersten Moment beruhigen. Jedoch weisen qualitative Befragungen darauf hin, dass es sich bei diesen Behelfs-Konstrukten oftmals um prekäre Unterkünfte handelt. Frietsch und Holbach stellen in ihrer Studie heraus, dass insbesondere bei „Helfern“ von wohnungslosen Frauen die Grenzen zwischen Freund, Zufallsbekanntschaft und Freier oftmals fließend sind (Frietsch und Holbach 2016, S. 17). Von einem stabilen festen Rückzugsort kann hier kaum gesprochen werden. Zudem sind die Wohnungslosen aufgrund ihres vorwiegenden Aufenthalts in Privatwohnungen viel weniger für die Hilfsangebote sichtbar und somit schwerer zu erreichen.

In einem zweiten Schritt werden die Aufenthaltsorte der Befragten in ihren Straßenepisoden näher betrachtet. Es wurde versucht, die Pendelbewegungen zwischen verschiedenen Aufenthaltsorten und den in der Literatur oft beschriebenen Wechsel zwischen Wohnungs- und Obdachlosigkeit, abzubilden. Für jede Straßenepisode konnten mehrere Aufenthaltsorte angegeben werden, wobei zusätzlich gefragt wurde, wo sie sich hauptsächlich aufgehalten haben. Jedoch haben die Befragten zumeist nur einen Aufenthaltsort angegeben (47,5\% bezogen auf die aktuelle Episode; $66,7 \%$ bezogen auf zurückliegende Episoden). So kann das oft beschriebene Pendeln der Betroffenen zwischen vielen verschiedenen Aufenthaltsorten so in den Daten nicht nachgewiesen werden.

Dies könnte mehrere Gründe haben, z. B. können die Betroffenen sich zwar stets bei wechselnden Freunden aufgehalten haben, in der Erhebung wird dies jedoch nicht deutlich, weil nur „Freunde“ als Aufenthaltsort angegeben wurde. Weiterhin kann es sein, dass zwar viele verschiedene Wechsel stattgefunden haben, die Befragten sich aber im Nachhinein an die vielen Wechsel gar nicht mehr erinnern können und demnach nur wenige zentrale Aufenthaltsorte angeben. Und schließlich kann ein Auslassen von Aufenthaltsorten auch eine Strategie gewesen sein, die Befragung abzukürzen.

Im Folgenden werden die genannten Hauptaufenthaltspunkte zum Befragungszeitpunkt analysiert. Am häufigsten wurden „Freunde“ (27,2\%), gefolgt von der „Straße“ mit 16,5\% genannt. Danach folgen Hilfeeinrichtungen $(13,8 \%)$ und Wohnung/WG-Zimmer $(13,4 \%)$. Alle anderen Aufenthaltsorte wie Notunterkunft, Eltern, Verwandte, Partner, Hotel/Hostel/Pension, Behelfsunterkunft, betreutes Wohnen oder Kinderheim spielen nur eine untergeordnete Rolle.

Weiterhin wurden die Aufenthaltsorte aller zurückliegenden Episoden gemeinsam betrachtet. Dies bedeutet für die Interpretation, dass es sich hierbei nicht, wie bei der vorherigen Betrachtung, um die Merkmalsausprägung unter den Befragten zum Erhebungszeitpunkt handelt, sondern dass die Verteilung angibt, wie häufig bestimmte Aufenthaltsorte in den Episoden genannt wurden. 
Hierbei wird als Aufenthaltsort mit 19,4\% am häufigsten die Straße genannt, dicht gefolgt von den Eltern mit 18,7\% und Freunden mit 17,9\%. Weiterhin häufig genannt wurde mit 9,5\% Wohnung/WG-Zimmer und mit 9,9\% betreutes Wohnen.

Auffällig ist, dass sich eine hohe Anzahl von Nennungen in den letzten zwei Jahren auf Aufenthaltsorte beziehen, die einen festen Wohnsitz darstellen, also beispielsweise „Eltern“ oder auch eine „eigenen Wohnung/WG“. Feste Wohnsitze scheinen somit in den letzten zwei Jahren für den Großteil der Befragten von zentraler Bedeutung gewesen zu sein. Da lediglich die Aufenthaltsorte, aber nicht die Aufenthaltsdauer an den jeweiligen Orten abgefragt wurde, kann jedoch nicht geschlussfolgert werden, dass sich die Betroffenen in den letzten zwei Jahren überwiegend an einem festen Wohnsitz aufgehalten haben.

Mit Blick auf die Aufenthaltsorte lässt sich ausgehend von der quantitativen Befragung der Betroffenen festhalten, dass „Straßenjugendliche“ überwiegend wohnungslos sind und als hauptsächlicher Aufenthaltsort Freunde genannt werden. Allerdings spielt auch Obdachlosigkeit, also das tatsächliche Leben ohne jegliche Unterkunft auf der Straße, eine nicht zu vernachlässigende Rolle. Hinsichtlich des Alters und des Geschlechts zeigen sich bei den Aufenthaltsorten keine markanten Unterschiede.

\subsection{Volljährigkeit verschärft Straßenkarrieren}

Um die Unterschiede in den Lebensbedingungen ohne festen Wohnsitz von minderjährigen Straßenjugendlichen und jungen Volljährigen herauszuarbeiten, wird zunächst die Altersstruktur der Befragten dargestellt (Abb. 1). Das ausgewiesene Alter bezieht sich dabei auf die aktuelle oder die letzte Straßenepisode. Für die Fälle, die zum Befragungszeitpunkt einen festen Wohnsitz hatten, wurde das Alter aus der Episode verwendet, in der sie zum letzten Mal ohne festen Wohnsitz waren.

Der Großteil der Befragten war mit 75,0\% (222 Befragte) während der aktuellen oder letzten Straßenepisode bereits volljährig. Der Anteil an Minderjährigen in der Stichprobe ist mit 25,0\% (74 Befragte) aber nicht unerheblich. Vierzehn Jahre oder jünger und somit Kinder waren 5,1\% der Befragten. Dies deckt sich mit Studien, die von wohnungslosen Kindern in Deutschland als Ausnahme ausgehen (Flick und Röhnsch 2009, S. 49; Hansbauer 1998), so dass der Begriff Straßenjugendliche gegenüber dem Begriff Straßenkinder als der angemessenere gesehen werden kann.

In der Befragungsgruppe sind junge Mädchen früher auf die Straße gelangt als junge Männer, die vor allem als junge Erwachsene wohnungs- oder obdachlos sind. Ausgehend von den Daten verschwindet dieser Unterschied allerdings, wenn man sich das Eintrittsalter in eine Straßenkarriere anschaut. Es liegt für beide Geschlechter bei 16 bis 17 Jahren.

Vielmehr scheint das Erreichen der Volljährigkeit einen erheblichen Einfluss auf die Lebenssituation zu haben, denn in den analysierten Daten ist ein sprunghafter Anstieg für die jungen Volljährigen festzustellen. Der Übertritt in die Volljährigkeit scheint somit prekäre Situationen entstehen zu lassen bzw. schon bestehende prekäre Situationen weiter zu verschlimmern.

Ein Aspekt, der in anderen Studien herausgearbeitet wurde und der in diesem Zusammenhang relevant sein könnte ist, dass die Jugendhilfe zumeist mit 18 Jahren 


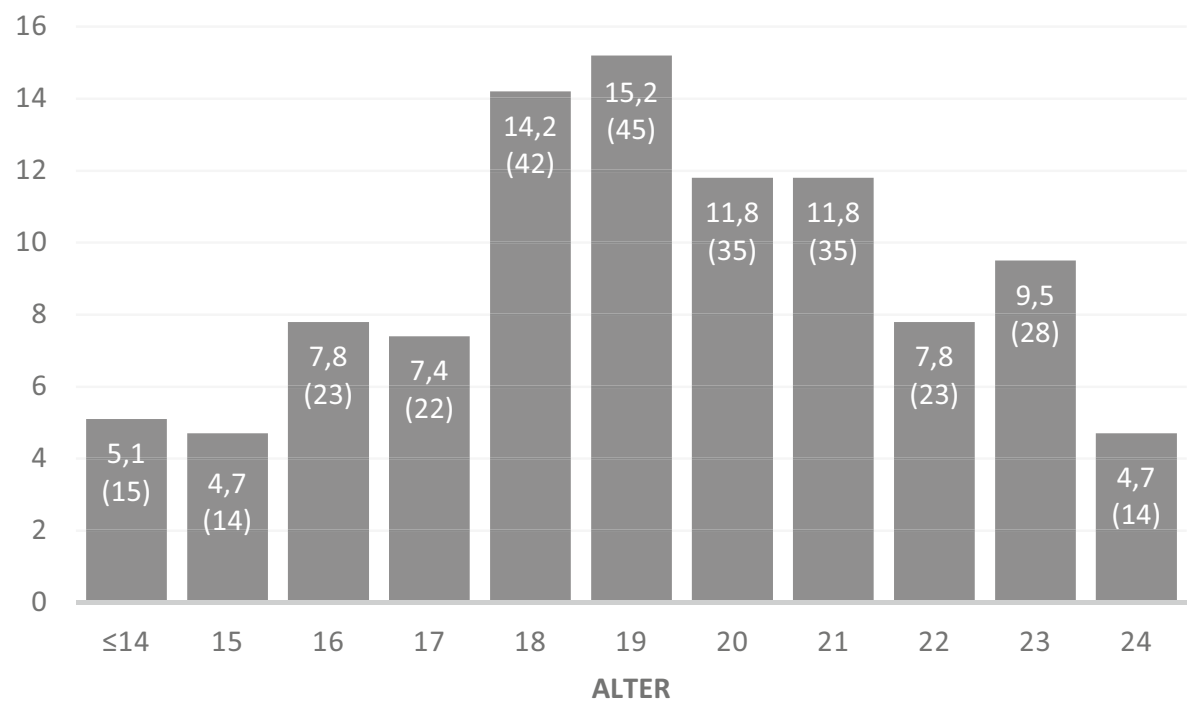

Abb. 1 Altersverteilung $(N=296)$

endet (Fendrich et al. 2016) und oft nur ein ungenügender Übergang in andere Hilfestrukturen (Arbeitsförderung, Grundsicherung, Sozialhilfe) erfolgt (Gerdes et al. 2016, S. 138).

Aus den Daten (Tab. 1) kann herausgelesen werden, dass eine Unterstützung des Jugendamts tatsächlich zumeist mit der Volljährigkeit endet. So geben zwei Drittel der unter 18-Jährigen an, Kontakt zum Jugendamt zu haben, zwischen dem 18. und 20. Lebensjahr sagen dies nur noch $14 \%$ und mit über 20 Jahren hat keiner mehr Kontakt zum Jugendamt. Dagegen nimmt das Jobcenter eine wichtigere Rolle ein. Ab dem 18. Lebensjahr haben hier über $70 \%$ Kontakt.

Aus qualitativen Untersuchungen wurde deutlich, dass dies nicht immer unproblematisch ist. Häufig fühlen sich die Jugendlichen von Mitarbeitenden der Jugendhilfe, vor allem der Jugendsozialarbeit gut unterstützt, haben Vertrauen zu den Kontakt-

Tab. 1 Kontakt zu Jugendamt und Jobcenter. Darstellung: Spaltenprozent (Anzahl)

\begin{tabular}{lllllllll}
\hline \multicolumn{3}{l}{ Kontakt zum Jugendamt } & \multicolumn{7}{c}{ Kontakt zum Jobcenter } \\
& $<18$ & $18-20$ & $>20$ & Gesamt & $<18$ & $18-20$ & $>20$ & Gesamt \\
\hline Nein & 36,1 & 86,1 & 100 & 75,7 & 54,8 & 29,6 & 29,3 & 33,6 \\
& $(26)$ & $(87)$ & $(74)$ & $(187)$ & $(23)$ & $(34)$ & $(29)$ & $(86)$ \\
Ja & 63,9 & 13,9 & 0 & 24,3 & 45,2 & 70,4 & 70,7 & 66,4 \\
& $(46)$ & $(14)$ & $(0)$ & $(60)$ & $(19)$ & $(81)$ & $(70)$ & $(170)$ \\
Gesamt & 100 & 100 & 100 & 100 & 100 & 100 & 100 & 100 \\
& $(72)$ & $(101)$ & $(74)$ & $(247)$ & $(42)$ & $(115)$ & $(99)$ & $(256)$ \\
Chi & & & & & $10,1 * *$ & & & \\
\hline
\end{tabular}

Signifikanzniveau: ${ }^{+} 0,1 ; * 0,05 ; * * 0,01 ; * * * 0,001$ 
personen aufgebaut, von anderen Behörden, wie dem Jobcenter sehen sie sich nicht selten zum „Fall““ degradiert (Beierle 2017; Mögling et al. 2015).

Die Hinwendung zum Jobcenter zeigt sich innerhalb der untersuchten Daten auch darin, dass die jungen Volljährigen bei der Frage ihrer Finanzierung am häufigsten SGB-II-Leistungen angegeben haben. Minderjährige Straßenjugendliche gaben dagegen in erster Linie den legalen Erwerb auf der Straße sowie Unterstützungsleistungen von Privatpersonen an.

\subsection{Unzufriedenheit mit dem Leben ohne festen Wohnsitz und positiver Blick in die Zukunft}

Ein weiterer wichtiger Aspekt bei der quantitativen Befragung Straßenjugendlicher waren die Gründe für ihre Situation. Bei der Auswertung zeigt sich, dass bei den Gründen für die Straßenkarriere sowohl bei den Minderjährigen als auch bei den jungen Volljährigen die familiären Problemlagen dominieren. Dies deckt sich mit Befunden vorangegangener Studien (Bodenmüller und Piepel 2003, S. 11 f.; Hansbauer et al. 1997). Ursächlich können hierbei verschiedene Familienkonstellationen (z.B. Patchwork-Familien), physische, psychische und emotionale Gewalt im Elternhaus (z. B. Missbrauch oder Vernachlässigung) oder sozialstrukturelle Gegebenheiten (z. B. Armut) sein (Flick und Röhnsch 2009, S. 49).

Mehr als die Hälfte der befragten Straßenjugendlichen ist mit ihrer Situation ohne festen Wohnsitz nicht zufrieden (gewesen) (30,5\% eher unzufrieden, 21,7\% sehr unzufrieden). Knapp ein Drittel kann sich nicht eindeutig zuordnen, und der Anteil derer, die mit ihrem Straßenleben sehr zufrieden sind, ist mit 7,0\% äußerst gering. Die teilweise in der Öffentlichkeit vertretene Meinung, dass sich viele Betroffene bewusst den Institutionen entziehen, um auf der Straße alternative Lebensentwürfe zu leben, kann somit nicht bestätigt werden. Nur etwa jeder zehnte volljährige Straßenjugendliche gab die „Suche nach mehr Freiraum“ als Hauptgrund für die Straßenkarriere an, bei den minderjährigen Jugendlichen traf dies nur auf einen Fall $\mathrm{zu}$.

Zum Vergleich wurde die Einschätzung zur Lebenssituation von jenen Befragten herangezogen, die zum Befragungszeitpunkt bereits einen festen Wohnsitz hatten und ihre Notsituation beenden konnten. Bei diesen Jugendlichen ist eine deutlich stärkere Zufriedenheit mit der aktuellen Situation zu erkennen. Die Hälfte der ehemals Wohnungs- oder Obdachlosen gibt an, mit ihrer aktuellen Situation zufrieden zu sein: 32,2\% sind eher zufrieden und 26,2\% sogar sehr zufrieden. Ein statistischer Zusammenhang zwischen der Zufriedenheit mit der aktuellen Situation und dem aktuellen Wohnstatus kann durch einen $\mathrm{Chi}^{2}$-Unabhängigkeitstest bestätigt werden.

Ein Großteil der Jugendlichen, die zum Zeitpunkt der Befragung keinen festen Wohnsitz hatten, glaubt, dass sich ihre Situation in den nächsten 12 Monaten maßgeblich positiv verändern wird. Etwa drei Viertel gehen davon aus, dass eine positive Veränderung eintreten wird (41,6\% eher ja, 33,5\% voll und ganz). Lediglich 4,3\% glauben, dass sich überhaupt nichts ändert, und 3,8\% glauben, dass sich eher nichts verändert. 16,8\% antworten mit ,teils/teils“.

Abgefragt wurde ebenfalls, was sich laut den Straßenjugendlichen in den nächsten 12 Monaten verändern wird. Diese Frage wurde als teilstandardisierte Multiple- 
Choice-Frage gestellt. Von den Jugendlichen, die zum Zeitpunkt der Befragung ohne festen Wohnsitz waren, wurde mit Abstand mit 76,1\% am häufigsten geäußert, dass sich die Wohnsituation ändern wird, das heißt, dass sie einen festen Wohnsitz finden werden. Am zweithäufigsten wurde eine Verbesserung der finanziellen Situation aufgezählt. Hierunter können Aspekte wie das Abbezahlen von Schulden als auch die Sicherstellung eines geregelten und festen Einkommens vermutet werden. 39-mal $(18,7 \%)$ wurde das Beginnen oder Beenden einer Ausbildung bzw. eines Studiums genannt, und 34-mal (16,3\%) wurde das Beginnen, Beenden oder Nachholen eines Schulabschlusses aufgezählt.

Somit ist festzustellen, dass die befragten Straßenjugendlichen zwar mit ihrer aktuellen Situation nachvollziehbarerweise eher nicht zufrieden sind, jedoch alles in allem positive Erwartungen an die Zukunft haben. Vor allem hinsichtlich einer positiven Entwicklung hin zu einem festen Wohnsitz scheinen die Jugendlichen optimistisch.

Dass andere Faktoren deutlich seltener genannt wurden, könnte sich dadurch erklären lassen, dass das Vorhandensein eines festen Wohnsitzes den Grundstein für weitere Veränderungen darstellt und erst durch die Absicherung einer Unterkunft neue Aspekte, wie z.B. Bildung, in den Vordergrund rücken.

\subsection{Insbesondere Obdachlose fühlen sich als ,Straßenjugendliche“}

In der DJI-Studie wurde, wie bereits erörtert, für junge Menschen ohne festen Wohnsitz die Bezeichnung „Straßenjugendliche“ gewählt. Darunter wurden sowohl obdach- als auch wohnungslose junge Menschen verstanden. Um bei der Untersuchung zu vermeiden, dass es zu Unklarheiten hinsichtlich der Zielgruppe der Erhebung kommt, wurde die Arbeitsdefinition (Abschnitt „Hintergrund der Studie“) bei der Interviewschulung aber auch bei der Fragebogengestaltung stets eingebunden. Somit sollte sichergestellt werden, dass alle jungen Menschen ohne festen Wohnsitz Zielgruppe der Erhebung sind.

Um zu überprüfen, ob und für wen der Begriff „Straßenjugendliche“ gerechtfertigt ist, wurde für jede Straßenepisode gefragt, ob sie sich als ,Straßenjugendliche/r" gefühlt haben. Dies erfolgte auch für Episoden mit festem Wohnsitz, um nachzuvollziehen, ob eine Identifizierung auch langfristig nachwirkt bzw. ob sich die Zuordnung zum Begriff ,Straßenjugend“ mit Aufnahme eines festen Wohnsitzes ändert.

Hinsichtlich der aktuellen bzw. letzten Straßenepisode zeigt sich, dass sich der Großteil der Befragten mit 23,3\% (67 Personen) eher und mit 28,8\% (83 Personen) voll und ganz als Straßenjugendliche fühlen. Obdachlose verstehen sich mit $53,2 \%$ (50 Personen) aber sehr viel häufiger voll und ganz als „Straßenjugendliche“ als wohnungslose junge Menschen, bei denen nur 16,9\% (32 Personen) sich mit dem Begriff identifizieren. Wohnungslose antworten mit 34,9\% (66 Personen) am häufigsten mit teils/teils. Hinsichtlich des Alters zeigen sich keine Unterschiede. Volljährige fühlen sich der Gruppe im gleichen Maße zugehörig wie Minderjährige.

Auch bei der Analyse der aktuellen Wohnsituation ergibt sich ein ähnliches Bild. Bei dieser Betrachtungsweise stand nicht mehr die aktuelle oder letzte Straßenepisode im Vordergrund, sondern die Einschätzung wurde anhand der Wohnsituation 
zum Befragungszeitpunkt analysiert. Somit fließen bei dieser Betrachtung nicht nur Befragte in die Analyse mit ein, die zum Befragungszeitpunkt ohne festen Wohnsitz waren, sondern auch diejenigen, die einen festen Wohnsitz hatten. Dies ermöglicht es, die beiden Gruppen hinsichtlich ihrer Einschätzung miteinander zu vergleichen. Insgesamt antworten hier die meisten mit 32,0\% (93 Personen), dass sie sich ,teils/ teils“" als Straßenjugendliche/r fühlen. Auch hier sieht sich der Großteil der Obdachlosen, nämlich 44,6\% (25 Personen), voll und ganz als Straßenjugendliche. Dahingehend sehen sich nur 14,7\% (22 Personen) der Wohnungslosen voll und ganz als Straßenjugendliche. Auch hier ist der Großteil der wohnungslosen Befragten eher unentschlossen, 38,0\% (57 Personen) antworten mit „teils/teils“.

Interessant ist jedoch auch die Einschätzung beim festen Wohnsitz, denn wider Erwarten würden sich 30,6\% (26 Personen), ,teils/teils“ nach wie vor als ,,Straßenjugendliche“ bezeichnen. 24,7\% (21 Personen) sehen sich nicht mehr als solche. Dass ein Großteil in dieser Frage unentschlossen ist, könnte damit zusammenhängen, dass die Betroffenen noch nicht lange mit festem Wohnsitz sind und sich somit möglicherweise immer noch der Gruppe der „Straßenjugendlichen“ zugehörig fühlen bzw. sich (noch) nicht sicher sind, ob diese Phase schon abgeschlossen ist.

Zusammenfassend kann, ausgehend von den zugrundeliegenden quantitativen Daten, demnach festgehalten werden, dass der Begriff „Straßenjugendliche“ tatsächlich bestimmte Assoziationen weckt. Vor allem Obdachlose fühlen sich von dem Begriff angesprochen. Somit scheint sich zu bestätigen, dass mit dem Begriff „Straße“ vor allem das gänzliche Fehlen einer Unterkunft und das direkte Leben auf der Straße verbunden wird. Eine genaue Spezifizierung, wer alles darunter zu verstehen ist, scheint somit sinnvoll, denn Wohnungslose können sich nur teilweise mit dem Begriff identifizieren. Hinsichtlich des Alters hat sich jedoch kein Effekt gezeigt, sowohl Minderjährige als auch Volljährige fühlen sich von der Bezeichnung angesprochen. Der Wortteil „Jugendliche“ scheint für die Gruppe der bis zu einschließlich 24-Jährigen unproblematisch zu sein.

\section{Reflexion der Studie und ihrer Ergebnisse}

Mit Blick auf die beschriebenen Herausforderungen von Forschung zu jungen Menschen ohne festen Wohnsitz sollen die Studie und die Ergebnisse reflektiert und der Mehrwert der Studie herausgearbeitet werden.

Der Zugang zur Zielgruppe erwies sich unproblematischer als erwartet. Insgesamt konnte eine Vielzahl an Jugendlichen und jungen Erwachsenen für die Befragung gewonnen werden. Hierbei spielte sicherlich die Unterstützung durch die Einrichtungen eine entscheidende Rolle. Vertrauen konnte dadurch aufgebaut werden, dass der Fragebogen vorab vor Ort vorgestellt wurde und ein Teil der dort arbeitenden Sozialarbeiterinnen und Sozialarbeiter nach einer Interviewschulung selbst die Interviews durchführten. Ebenso erhielten die befragten Jugendlichen für die Teilnahme an der ca. 30-minütigen Befragung $20 €$ als Incentive, was ein erheblicher monetärer Anreiz war.

Die weite Definition, die „Straßenjugendliche“ als junge Menschen bis 24 Jahren versteht, welche wohnungslos oder obdachlos sind, hat sich für diese Studie 
bewährt. Auch wenn sich die spezifischen Pendelbewegungen junger Menschen zwischen Wohnungs- und Obdachlosigkeit und zwischen den dazugehörigen Orten auf Grundlage der Daten nicht statistisch abbilden lassen, zeigt sich doch, dass die Straßenkarrieren sowohl durch Wohnungs- als auch durch Obdachlosigkeit geprägt sind. Dass obdachlose Jugendliche sich eher mit dem Begriff identifizieren als wohnungslose Jugendliche, es zwischen Minderjährigen oder Volljährigen aber keinen Unterschied gibt, lässt den Begriff „Straßenjugendliche“ plausibel erscheinen.

Unter anderem wegen des Zugangs über Einrichtungen muss bei der Interpretation der Einzelergebnisse stets reflektiert werden, dass es $\mathrm{zu}$ Verzerrungen in der Erhebung gekommen sein kann. Dennoch ist es durch die quantitative Studie erstmals gelungen, einen Einblick in die Lebenssituation zahlreicher wohnungsloser und obdachloser Jugendlicher zu erhalten. Viele zuvor qualitativ gewonnenen Forschungsbefunde konnten auf Basis der Datengrundlage quantitativ untermauert werden. Straßenkarrieren sind demnach in erheblichem Ausmaß durch Wohnungslosigkeit geprägt. Zwar haben diese Jugendlichen einen behelfsmäßigen Unterschlupf gefunden, die Straße als Aufenthaltsort spielt aber für manche dennoch eine Rolle. Auf der anderen Seite ist aber zu vermuten, dass es eine große Dunkelziffer an Personen gibt, die wohnungslos sind, nicht im öffentlichen Raum sichtbar sind und zugleich keinen sicheren Rückzugsort haben. Dies stellt die verschiedenen Hilfesysteme vor die Herausforderung, Angebote und Ideen zu entwickeln, um diese Personengruppe mit ihren Angeboten besser erreichen zu können.

Mit Erreichen der Volljährigkeit nimmt der Anteil derer, die in Kontakt zum Jugendamt stehen, ab, wohingegen das Jobcenter die zentrale Anlaufstelle bildet. Somit konnte die Volljährigkeit als neuralgischer Punkt in den Ergebnissen herausgearbeitet werden. Auf diese Schwierigkeiten beim Übergang in andere Leistungsbezüge, Beendigung von Hilfen etc. ist in anderen Studien immer wieder verwiesen worden (Mögling et al. 2015; Skrobanek et al. 2010). Die Studienergebnisse können aber nicht klar zeigen, was hier Ursache und was Wirkung ist und wie die Hilfeprozesse in den jeweiligen Fällen verlaufen sind.

Deutlich wird aber, dass sich die Lebenssituation von minderjährigen und jungen volljährigen Straßenjugendlichen unterscheidet, was mit der Möglichkeit der eigenständigen Inanspruchnahme von Hilfen und damit, dass sie nicht mehr in Obhut genommen und gegen ihren Willen untergebracht werden dürfen, begründet sein dürfte.

\section{Ausblick}

Ziel der Untersuchung war es, einen Einblick in die Lebenssituation junger Menschen ohne festen Wohnsitz zu erhalten. Mit einer Anzahl von rund 300 Befragten konnte dafür eine solide Datenbasis hergestellt werden.

Auf dieser Grundlage konnte jedoch eine Frage, aus Politik und Fachöffentlichkeit, nicht beantwortet werden: Wie viele junge Menschen sind in Deutschland wohnungs- bzw. obdachlos?

Um sich dieser Frage anzunehmen, wurde im Anschluss an die Befragung der Jugendlichen eine deutschlandweite Onlinebefragung von Fachkräften durchgeführt 
(Hoch 2017). Durch die Fachkräftebefragung und eine anschließende Schätzung und Hochrechnung der Ergebnisse konnte eine Anzahl von 37.000 Jugendlichen und jungen Erwachsenen ohne festen Wohnsitz in Deutschland ermittelt werden. Die Altersgrenze wurde in der 2. Projektphase angehoben. Die ermittelte Anzahl an Betroffenen bezieht sich auf junge Menschen bis einschließlich 26 Jahren, um alle Personen, die nach dem Kinder- und Jugendhilfegesetz Anspruch auf Hilfen haben können, mit einzuschließen.

Die 1. Projektphase war für die Schätzung des Ausmaßes der Anzahl „Straßenjugendlicher" in der 2. Projektphase relevant, um einen Einblick in die Lebenswelt der jungen Menschen zu erhalten und darauf aufbauend eine Definition entwickeln zu können, die für die Fachkräftebefragung herangezogen werden kann. Letztlich hat es sich jedoch herausgestellt, dass eine breite Betrachtung von ,Straßenjugend“, welche wohnungslose und obdachlose Jugendliche miteinschließt, das Phänomen am besten beschreibt. Bis auf die Ausweitung der Altersgrenzen, waren keine Anpassungen an der Arbeitsdefinition der 1. Phase notwendig.

$\mathrm{Zu}$ berücksichtigen ist jedoch, dass es sich bei der Hochrechnung der Anzahl „Straßenjugendlicher“" nur um eine Momentaufnahme handelt. Nicht möglich sind somit Aussagen darüber, inwiefern sich die Anzahl betroffener Jugendlicher in den letzten Jahren verändert hat. Hierzu müsste eine solche Erhebung in regelmäßigen Abständen wiederholt werden. Möglicherweise bietet die geplante Wohnungslosenstatistik der Bundesregierung einen Ansatzpunkt, um Entwicklungen und Veränderungen bei der Gruppe von jungen Menschen, die ohne festen Wohnsitz sind, im Zeitverlauf analysieren zu können.

Funding Open Access funding enabled and organized by Projekt DEAL.

Open Access Dieser Artikel wird unter der Creative Commons Namensnennung 4.0 International Lizenz veröffentlicht, welche die Nutzung, Vervielfältigung, Bearbeitung, Verbreitung und Wiedergabe in jeglichem Medium und Format erlaubt, sofern Sie den/die ursprünglichen Autor(en) und die Quelle ordnungsgemäß nennen, einen Link zur Creative Commons Lizenz beifügen und angeben, ob Änderungen vorgenommen wurden.

Die in diesem Artikel enthaltenen Bilder und sonstiges Drittmaterial unterliegen ebenfalls der genannten Creative Commons Lizenz, sofern sich aus der Abbildungslegende nichts anderes ergibt. Sofern das betreffende Material nicht unter der genannten Creative Commons Lizenz steht und die betreffende Handlung nicht nach gesetzlichen Vorschriften erlaubt ist, ist für die oben aufgeführten Weiterverwendungen des Materials die Einwilligung des jeweiligen Rechteinhabers einzuholen.

Weitere Details zur Lizenz entnehmen Sie bitte der Lizenzinformation auf http://creativecommons.org/ licenses/by/4.0/deed.de.

\section{Literatur}

Alleweldt, E., \& Leuschner, V. (2004). Freundschaften auf der Straße. Berliner Journal für Soziologie, 14(3), 339-356.

Beierle, S. (2017). Praxisbericht zur Projektarbeit mit Straßenjugendlichen. Erkenntnisse aus den Modellprojekten des Innovationsfonds (des Kinder- und Jugendplans) im Bereich Jugendsozialarbeit (2014-2016). München: Deutsches Jugendinstitut. 
Beierle, S. (2019). Projekte für die Zielgruppe der Straßenjugendlichen entwickeln und durchführen. Erkenntnisse aus Modellprojekten des Innovationsfonds des Bundes im Handlungsfeld Jugendsozialarbeit (2014-2016) und (2017-2019) (Handreichung). Halle (Saale): Deutsches Jugendinstitut.

Bielert, D. (2006). Straßenkarrieren von Kindern und Jugendlichen. Wenn es passiert ist ... Erklärungen aus Sicht der Jugendlichen und Hilfestellung für ihre Eltern. Dissertation. Hamburg: Universität Hamburg, Fachbereich Psychologie. https://ediss.sub.uni-hamburg.de/bitstream/ediss/1484/1/ Bielertdissertation.pdf

Bodenmüller, M., \& Piepel, G. (2003). Streetwork und Überlebenshilfen. Entwicklungsprozesse von Jugendlichen aus Straßenszenen (1. Aufl.). Beltz-Votum. Weinheim: Beltz.

Brändle-Stöh, M. (1999). Was braucht der Mensch zum Wohnen? Anmerkungen zum Wohn-Bedarf aus der Sicht einer allgemeinen Theorie menschlicher Bedürfnisse. SozialAktuell, 9, 16-23.

Bundesministerium für Familie, Senioren, Frauen und Jugend. (2017). 15. Kinder- und Jugendbericht. Bericht über die Lebenssituation junger Menschen und die Leistungen der Kinder- und Jugendhilfe in Deutschland ; Stellungnahme der Bundesregierung zum Bericht der Sachverständigenkommission ; Bericht der Sachverständigenkommission (Drucksache/Deutscher Bundestag, 18. Wahlperiode, 18/11050, Stand: Februar 2017, 1. Auflage). Berlin.

Busch-Geertsema, V., Evers, J., \& Ruhstrat, E.-U. (2016). Prävention von Wohnungslosigkeit in Nordrhein-Westfalen. Ergebnisse einer landesweiten Untersuchung. In S. Gillich \& R. Keicher (Hrsg.), Suppe, Beratung, Politik. Anforderungen an eine moderne Wohnungsnotfallhilfe (1. Aufl. S. 111-127). Wiesbaden: Springer VS.

Destatis (Hrsg.). (2020). Gut ein Viertel der 25-Jährigen wohnte 2019 noch im Haushalt der Eltern. Pressemitteilung: Nr. N045. https://www.destatis.de/DE/Presse/Pressemitteilungen/2020/08/PD20_N045_ 122.html. Zugegriffen: 2. Juni 2021.

Die Bundesregierung Statistik zu Wohnungslosigkeit. https://www.bundesregierung.de/breg-de/suche/ wohnungslose-in-deutschland-1672730. Zugegriffen: 7. Juni 2021.

Fendrich, S., Pothmann, J., \& Tabel, A. (2016). Monitor Hilfen zur Erziehung 2016. Dortmund: Eigenverlag Forschungsverbund DJI/TU Dortmund.

Fernandez, K. (2014). Wechselbeziehungen zwischen gesellschaftlicher Sicherheitsorientierung und den Straßenkarrieren Jugendlicher und junger Erwachsener. Österreich Z Soziol, 39, 325-340.

Flick, U., \& Röhnsch, G. (2009). Jugendobdachlosigkeit. Sozial Extra, 33(5-6), 49-52.

Frietsch, R., \& Holbach, D. (2016). Lebensorientierung für junge Wohnungslose. LZG-Modellprojekt. Landeszentrale für Gesundheitsförderung in Rheinland-Pfalz.

Gerdes, B., Steding, U., \& Würfel, G. (2016). Junge Menschen in (Wohnungs-)Not. In S. Gillich \& R. Keicher (Hrsg.), Suppe, Beratung, Politik. Anforderungen an eine moderne Wohnungsnotfallhilfe (1. Aufl. S. 131-140). Wiesbaden: Springer VS.

Hansbauer, P. (Hrsg.). (1998). Kinder und Jugendliche auf der Straße. Analysen, Strategien, Lösungsansätze. Münster: Votum.

Hansbauer, P., Permien, H., \& Zink, G. (1997). Gestern TrebegängerInnen - heute Straßenkinder? Gemeinsamkeiten und Unterschiede. neue praxis, 27, 395-412.

Häußermann, H., \& Siebel, W. (2000). Soziologie des Wohnens. Eine Einführung in Wandel und Ausdifferenzierung des Wohnens. Grundlagentexte Soziologie, Bd. 2. Weinheim: Juventa.

Hoch, C. (2016). Straßenjugendliche in Deutschland - eine Erhebung zum Ausmaß des Phänomens. Zwischenbericht - zentrale Ergebnisse der 1. Projektphase. Wissenschaftliche Texte. Halle (Saale): Deutsches Jugendinstitut.

Hoch, C. (2017). Straßenjugendliche in Deutschland - eine Erhebung zum Ausmaß des Phänomens. Endbericht - zentrale Ergebnisse der 2. Projektphase. München: Deutsches Jugendinstitut.

Hurrelmann, K., \& Quenzel, G. (2015). Lost in transition: status insecurity and inconsistency as hallmarks of modern adolescence. International Journal of Adolescence and Youth, 20(3), 261-270.

Liebel, M. (2018). Leichtfertige Rhetorik: „Straßenkinder“. Ein interessengeleiteter Kampfplatz. sozialmagazin, 43(1-2), 6-13.

Möbius, T. (1996). Lebensort Straße: Erste Einschätzungen aus den beteiligten Standorten „Hamburg - der lokale Kontext“". In Institut für soziale Arbeit e.V. (Hrsg.), Lebensort Straße - Kinder und Jugendliche in besonderen Problemlagen (S. 111-140). Münster: Votum.

Mögling, T., Tillmann, F., \& Reißig, B. (2015). Entkoppelt vom System. Jugendliche am Übergang ins junge Erwachsenenalter und Herausforderungen für Jugendhilfestrukturen. Eine Studie des Deutschen Jugendinstituts im Auftrag der Vodafone Stiftung Deutschland (Vodafone Stiftung Deutschland, Hrsg.)

Permien, H., \& Zink, G. (1998). Endstation Straße? Straßenkarrieren aus der Sicht von Jugendlichen. München: DJI. 
Reißig, B. (2019). Übergänge im Jugendalter - Alte Anforderungen, neue Herausforderungen?! Jugendhilfe, 57(4), 367-373.

Schaak, T. (2009). Obdachlose, auf der Straße lebende Menschen in Hamburg 2009. Eine empirische Untersuchung

Simon, T. (2006). Jugendliche auf der Straße. In M. D. Witte \& U. Sander (Hrsg.), Erziehungsresistent? „Problemjugendliche“ als besondere Herausforderung für die Jugendhilfe 2. Aufl. Grundlagen der sozialen Arbeit, (Bd. 15, S. 153-167). Baltmannsweiler: Schneider-Verl. Hohengehren.

Skrobanek, J., Mögling, T., \& Tillmann, F. (2010). Verlorene Jugendliche am Übergang Schule - Beruf. Empirische Befunde über junge „DropOuts“, 201(67), 41-64. 